\title{
AiMT
}

Advances in Military Technology

Vol. 15, No. 1, 2020, pp. 125-136

ISSN 1802-2308, eISSN 2533-4123

DOI 10.3849/aimt.01361

\section{Computational Reconstruction of an Anti-Aircraft Artillery Firing Scenario}

\author{
W. Bużantowicz* and D. Rodzik \\ Military University of Technology, Warsaw, Poland
}

\begin{abstract}
The manuscript was received on 8 October 2019 and was accepted after revision for publication as research paper on 21 March 2020.
\end{abstract}

\begin{abstract}
:
The paper describes a computational reconstruction of an anti-aircraft artillery firing scenario and its use in analysing firing data. Computational reconstruction supported by $3 D$ visualization can be a useful tool for evaluating soldiers' training. A system for collecting, analysing and visualising the results of anti-aircraft artillery firing is described. The information collected can then be re-used to evaluate the effectiveness of soldiers' training, and to make an optimal use of the available data. Particular attention is paid to the solution of the problem of restoring the trajectory of the target and the projectiles, and to their synchronisation in time.
\end{abstract}

\section{Keywords:}

acoustic locator, anti-aircraft artillery, computational methods, spline, visualisation

\section{Introduction}

The quality of artillery crews training has a large impact on the effectiveness of antiaircraft defence. The level of the training of these crews (subunits) during planned combat exercises at military training centres is a process that requires close monitoring, including evaluation procedures that are objective, immediate and precise. It is important that a proper mechanism is in place to verify the level of soldiers' training.

Military organisations evaluate anti-aircraft artillery firing using, among others, optical [1-3], televisual-optical [4], radar [5,6], and acoustic methods (for selected examples of systems see [7-9]).

The optical methods applied by Polish anti-aircraft defence troops employ handheld optical devices. These are subject to a high degree of dependence on the assessor's subjective judgement, as well as a number of other limitations, including the following factors:

\footnotetext{
* Corresponding author: Faculty of Mechatronics and Aerospace, Military University of Technology, Gen. S. Kaliskiego 2 Str., 00-908 Warsaw, Poland. Phone: +48 261839 452, E-mail: witold.buzantowicz@wat.edu.pl
} 
- the determination of the moment at which the projectile is at a minimum distance from the target is imprecise and depends on the person making the assessment,

- the evaluation expressed in thousandths is ambiguous,

- there is a lack of precision in the manual tracking of the target,

- observations are dependent on weather conditions.

Experience acquired over many years supports the conclusion that the optical evaluation methods used by Polish anti-aircraft defence troops are both unreliable (lacking in objectivity) and ineffective in their application of results (for example, results cannot be digitally recorded). This is the case not only in the evaluation of subunits undergoing training, but also in regard to further improvements of the skills of anti-aircraft crews (subunits) during barracks exercises.

Although radar methods can be highly precise, they require the use of complex and expensive tracking radars, and are therefore not cost-effective. Likewise, advanced televisual-optical systems with digital image processing also offer a relatively high precision, but are highly dependent on weather conditions.

Acoustic systems for the evaluation of artillery shots are used with increasing frequency. They effectively support the objective, which is fast and precise evaluations of combat action results, as well as allowing for real-time visual presentation and archiving. Acoustic systems are not oversensitive to variable weather conditions (including cloud cover). They can be used during both day and night exercises, and in all seasons. Acoustic systems operate according to the following two principles:

- every movement of the projectile is accompanied by a pressure disturbance in the medium in which it is travelling,

- the wave generated by the projectile's motion in an elastic medium is used as a source of information on the projectile's position relative to the acoustic measuring system.

Based on these principles, an acoustic miss-distance indicator (AMDI) system for evaluating aerial target shots has been developed at the Military University of Technology in conjunction with the Air Force Institute of Technology. It is intended to be used for:

- detection and determination of the coordinates of projectiles in flight relative to an aerial target,

- real-time visualisation of the results of firing at an aerial target,

- recording and analysis of the results of firing (qualitative, quantitative and statistical),

- collecting information required for evaluating the performance of artillery actions according to applicable rules.

In the proposed system, the aerial target is fitted with an acoustic locator (Fig. 1). While firing at the target, the locator detects pressure disturbances generated by the projectile, determines their parameters, and transmits the data by radio to a ground station, where they are processed by a CPU and displayed on a display screen. The results of firing are presented in real time at the firing position in the form of "bullet holes" on a virtual "shooting shield", which appear on a target disc resembling a plan position indicator. These results, presented in a simplified 2D form, do not fully exploit the potential of the gathered information. 


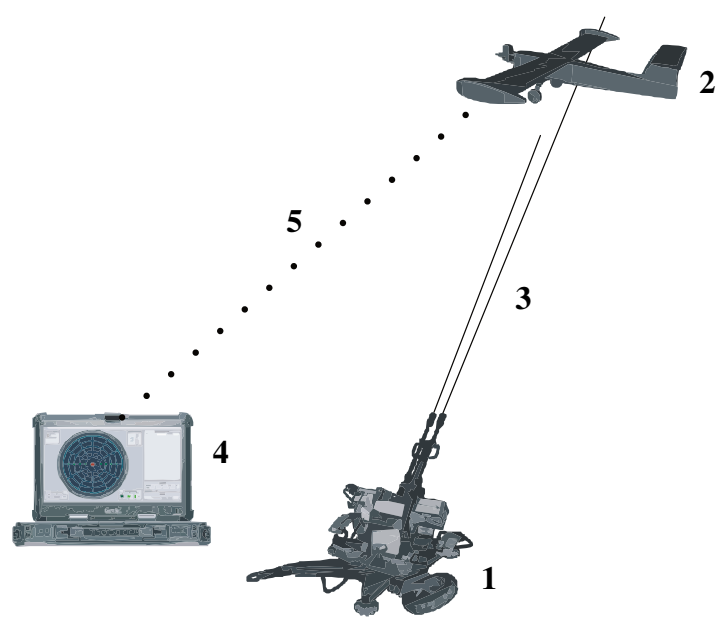

Fig. 1 Operating principles of the AMDI system:

1 - anti-aircraft cannon; 2 - aerial target with acoustic locator;

3 - projectile trajectories; 4 - ground unit; 5 - communication channel

However, the archiving of data from individual shots in the system database makes it possible to reprocess and analyse these data using a dedicated software tool in post-processing mode, based on mathematical models of simulation, analysis and statistics modules.

The main goal of the paper is to present the possibilities for using the proposed computational reconstruction to analyse anti-aircraft artillery firing data.

\section{Spline-Based Aerial Target Trajectory Reconstruction}

The fundamental idea behind the aerial target trajectory reconstruction is based on the definition of smooth curves through a number of points collected by an on-board GPS module. This allows the route to be reconstructed based on the known coordinates and velocities of the target in subsequent instants of time $t_{i}, i \in\{0,1,2, \ldots, n\}$. Moreover, it is a simple way to provide a space-time correlation with the recorded results of the anti-aircraft artillery fire.

The problem of spatial flight route description using splines is well known and widely described in the literature [10-15]. In our solution, we have decided to use the 3D quartic spline $s(t)$ of the form

$$
\boldsymbol{s}(t)=\boldsymbol{a}_{i} t^{4}+\boldsymbol{b}_{i} t^{3}+\boldsymbol{c}_{i} t^{2}+\boldsymbol{d}_{i} t+\boldsymbol{e}_{i}
$$

where

$$
\boldsymbol{a}_{i}=\left[\begin{array}{l}
a_{i x} \\
a_{i y} \\
a_{i z}
\end{array}\right], \quad \boldsymbol{b}_{i}=\left[\begin{array}{l}
b_{i x} \\
b_{i y} \\
b_{i z}
\end{array}\right], \quad \boldsymbol{c}_{i}=\left[\begin{array}{c}
c_{i x} \\
c_{i y} \\
c_{i z}
\end{array}\right], \quad \boldsymbol{d}_{i}=\left[\begin{array}{c}
d_{i x} \\
d_{i y} \\
d_{i z}
\end{array}\right], \quad \boldsymbol{e}_{i}=\left[\begin{array}{c}
e_{i x} \\
e_{i y} \\
e_{i z}
\end{array}\right]
$$


It is assumed that:

- $\boldsymbol{s}(t), \dot{\boldsymbol{s}}(t)$ and $\ddot{\boldsymbol{s}}(t)$ are continuous on the time interval $\left\langle t_{0}, t_{n}\right\rangle$,

- for each time interval $t \in\left\langle t_{i}, t_{i+1}\right\rangle, i \in\{0,1,2, \ldots, n-1\}$ the quartic spline is identical to the piecewise polynomial, i.e. $s(t) \equiv s_{i}(t)$,

- each of two subsequent piecewise polynomial functions are joined at the same data point, i.e. $\boldsymbol{s}_{i}\left(t_{i}\right)=\boldsymbol{s}_{i-1}\left(t_{i}\right)$,

- the first and the second derivatives of the spline polynomials are set to be equal at the position reference point, i.e. $\dot{s}_{i}\left(t_{i}\right)=\dot{s}_{i-1}\left(t_{i}\right), \ddot{s}_{i}\left(t_{i}\right)=\ddot{s}_{i-1}\left(t_{i}\right)$,

- $\ddot{s}_{n-1}\left(t=t_{n}\right)=\ddot{s}_{0}\left(t=t_{0}\right)=0$.

The set of coefficients for each of the piecewise polynomial functions can be found via the vector-matrix equation, constructed using Eq. (1) for $(n+1)$ knot points, and conditions defined above:

$$
\mathbf{f}=\mathbf{T} \Omega
$$

where $\boldsymbol{\Omega} \in \mathbb{R}^{5 n \times 3}$ is the matrix of piecewise polynomial coefficients, $\mathbf{T} \in \mathbb{R}^{5 n \times 5 n}$ is the power matrix of approximate polynomials and their derivatives given as

$$
\mathbf{T}=\left[\begin{array}{ccc}
\boldsymbol{\tau} & \dot{\boldsymbol{\tau}} & \ddot{\boldsymbol{\tau}}
\end{array}\right]^{\mathrm{T}}
$$

and $\mathbf{f} \in \mathbb{R}^{5 n \times 3}$ is the matrix collecting knot-point coordinates matrix $\mathbf{p}$, velocity constraints matrix $\overline{\mathbf{v}}$ and acceleration constraints matrix $\overline{\mathbf{w}}$

$$
\mathbf{f}=\left[\begin{array}{llll}
\mathbf{p} & \overline{\mathbf{v}} & \overline{\mathbf{w}} & \mathbf{0}^{1 \times 3}
\end{array}\right]^{\mathrm{T}}
$$

where $\mathbf{0}^{1 \times 3}$ means null matrix of size $1 \times 3$. In Eqs (4) and (5) specified matrices can be written in expanded forms as follows:

$$
\begin{aligned}
\boldsymbol{\tau} \in \mathbb{R}^{2 n \times 5 n}: \boldsymbol{\tau} & =\left[\begin{array}{cccc}
\boldsymbol{\tau}_{0} & \mathbf{0}^{1 \times 5} & \cdots & \mathbf{0}^{1 \times 5} \\
\boldsymbol{\tau}_{1} & \mathbf{0}^{1 \times 5} & \cdots & \mathbf{0}^{1 \times 5} \\
\mathbf{0}^{1 \times 5} & \boldsymbol{\tau}_{1} & \cdots & \mathbf{0}^{1 \times 5} \\
\mathbf{0}^{1 \times 5} & \boldsymbol{\tau}_{2} & \cdots & \mathbf{0}^{1 \times 5} \\
\vdots & \vdots & \ddots & \vdots \\
\mathbf{0}^{1 \times 5} & \mathbf{0}^{1 \times 5} & \cdots & \boldsymbol{\tau}_{n-1} \\
\mathbf{0}^{1 \times 5} & \mathbf{0}^{1 \times 5} & \cdots & \boldsymbol{\tau}_{n}
\end{array}\right], \quad \boldsymbol{\tau}_{i}=\left[\begin{array}{lllll}
t_{i}^{4} & t_{i}^{3} & t_{i}^{2} & t_{i} & 1
\end{array}\right] \\
\dot{\boldsymbol{\tau}} \in \mathbb{R}^{2 n \times 5 n}: \dot{\boldsymbol{\tau}}^{1 \times 5} & =\left[\begin{array}{cccc}
\dot{\boldsymbol{\tau}}_{0} & \mathbf{0}^{1 \times 5} & \cdots & \mathbf{0}^{1 \times 5} \\
\dot{\boldsymbol{\tau}}_{1} & \mathbf{0}^{1 \times 5} & \cdots & \mathbf{0}^{1 \times 5} \\
\mathbf{0}^{1 \times 5} & \dot{\boldsymbol{\tau}}_{1} & \cdots & \mathbf{0}^{1 \times 5} \\
\mathbf{0}^{1 \times 5} & \dot{\boldsymbol{\tau}}_{2} & \cdots & \mathbf{0}^{1 \times 5} \\
\vdots & \vdots & \ddots & \vdots \\
\mathbf{0}^{1 \times 5} & \mathbf{0}^{1 \times 5} & \cdots & \dot{\boldsymbol{\tau}}_{n-1} \\
\mathbf{0}^{1 \times 5} & \mathbf{0}^{1 \times 5} & \cdots & \dot{\boldsymbol{\tau}}_{n}
\end{array}\right], \quad \dot{\boldsymbol{\tau}}_{i}=\left[\begin{array}{lllll}
4 t_{i}^{3} & 3 t_{i}^{2} & 2 t_{i} & 1 & 0
\end{array}\right]
\end{aligned}
$$




$$
\begin{aligned}
& \ddot{\boldsymbol{\tau}} \in \mathbb{R}^{n \times 5 n}: \\
& \ddot{\boldsymbol{\tau}}=\left[\begin{array}{cccccc}
\ddot{\boldsymbol{\tau}}_{1} & -\ddot{\boldsymbol{\tau}}_{1} & \mathbf{0}^{1 \times 5} & \cdots & \mathbf{0}^{1 \times 5} & \mathbf{0}^{1 \times 5} \\
\mathbf{0}^{1 \times 5} & \ddot{\boldsymbol{\tau}}_{2} & -\ddot{\boldsymbol{\tau}}_{2} & \cdots & \mathbf{0}^{1 \times 5} & \mathbf{0}^{1 \times 5} \\
\vdots & \vdots & \vdots & \ddots & \vdots & \vdots \\
\mathbf{0}^{1 \times 5} & \mathbf{0}^{1 \times 5} & \mathbf{0}^{1 \times 5} & \cdots & -\ddot{\boldsymbol{\tau}}_{n-2} & \mathbf{0}^{1 \times 5} \\
\mathbf{0}^{1 \times 5} & \mathbf{0}^{1 \times 5} & \mathbf{0}^{1 \times 5} & \cdots & \ddot{\boldsymbol{\tau}}_{n-1} & -\ddot{\boldsymbol{\tau}}_{n-1} \\
\ddot{\boldsymbol{\tau}}_{0} & \mathbf{0}^{1 \times 5} & \mathbf{0}^{1 \times 5} & \cdots & \mathbf{0}^{1 \times 5} & -\ddot{\boldsymbol{\tau}}_{n}
\end{array}\right], \quad \ddot{\boldsymbol{\tau}}_{i}=\left[\begin{array}{lllll}
12 t_{i}^{2} & 6 t_{i} & 2 & 0 & 0
\end{array}\right] \\
& \mathbf{p} \in \mathbb{R}^{2 n \times 3}: \quad \mathbf{p}=\left[\begin{array}{lllllll}
\mathbf{p}_{0} & \mathbf{p}_{1} & \mathbf{p}_{1} & \cdots & \mathbf{p}_{n-1} & \mathbf{p}_{n-1} & \mathbf{p}_{n}
\end{array}\right]^{\mathrm{T}}, \quad \mathbf{p}_{i}=\left[\begin{array}{lll}
p_{i x} & p_{i y} & p_{i z}
\end{array}\right] \\
& \overline{\mathbf{v}} \in \mathbb{R}^{2 n \times 3}: \quad \overline{\mathbf{v}}=\left[\begin{array}{lllllll}
\overline{\mathbf{v}}_{0} & \overline{\mathbf{v}}_{1} & \overline{\mathbf{v}}_{1} & \cdots & \overline{\mathbf{v}}_{n-1} & \overline{\mathbf{v}}_{n-1} & \overline{\mathbf{v}}_{n}
\end{array}\right]^{\mathrm{T}}, \quad \overline{\mathbf{v}}_{i}=\left[\begin{array}{lll}
\bar{v}_{i x} & \bar{v}_{i y} & \bar{v}_{i z}
\end{array}\right] \\
& \overline{\mathbf{w}} \in \mathbb{R}^{(n-1) \times 3}: \quad \overline{\mathbf{w}}=\left[\begin{array}{llll}
\overline{\mathbf{w}}_{1} & \overline{\mathbf{w}}_{2} & \cdots & \overline{\mathbf{w}}_{n-1}
\end{array}\right]^{\mathrm{T}}, \quad \overline{\mathbf{w}}_{j}=\left[\begin{array}{lll}
\bar{w}_{j x} & \bar{w}_{j y} & \bar{w}_{j z}
\end{array}\right]
\end{aligned}
$$

where $i \in\{0,1,2, \ldots, n\}, j \in\{1,2, \ldots, n-1\}$, and $\mathbf{0}^{1 \times 5}$ is a null matrix of size $1 \times 5$.

Taking into account the above, the matrix $\boldsymbol{\Omega}$ is found by solving the rearranged system of Eq. (3)

$$
\mathbf{\Omega}=\mathbf{T}^{-1} \mathbf{f}
$$

to obtain the required coefficients of piecewise polynomial functions:

$$
\boldsymbol{\Omega}=\left[\begin{array}{llll}
\mathbf{\Omega}_{0} & \boldsymbol{\Omega}_{1} & \cdots & \boldsymbol{\Omega}_{n-1}
\end{array}\right]^{\mathrm{T}}, \quad \boldsymbol{\Omega}_{i}=\left[\begin{array}{lll}
a_{i x} & a_{i y} & a_{i z} \\
b_{i x} & b_{i y} & b_{i z} \\
c_{i x} & c_{i y} & c_{i z} \\
d_{i x} & d_{i y} & d_{i z} \\
e_{i x} & e_{i y} & e_{i z}
\end{array}\right]
$$

where $i \in\{0,1, \ldots, n-1\}$.

Finally, based on $\boldsymbol{\Omega}$, the set of $n$ polynomials is evaluated for each of the flat components $x(t), y(t)$ and $z(t)$ of the spatial spline $s(t)$.

For visualisation purposes, the aerial target is taken as a material point moving along a trajectory given by Eq. (1). Defining $\dot{\boldsymbol{s}}(t)$ and $\ddot{\boldsymbol{s}}(t)$ via the flat components $x(t), y(t)$ and $z(t)$, the following vectors are obtained:

$$
\dot{\boldsymbol{s}}(t)=\mathbf{v}=\left[\begin{array}{c}
v_{x} \\
v_{y} \\
v_{z}
\end{array}\right]=\left[\begin{array}{c}
\dot{x} \\
\dot{y} \\
\dot{z}
\end{array}\right], \quad \ddot{\boldsymbol{s}}(t)=\mathbf{w}=\left[\begin{array}{c}
w_{x} \\
w_{y} \\
w_{z}
\end{array}\right]=\left[\begin{array}{c}
\ddot{x} \\
\ddot{y} \\
\ddot{z}
\end{array}\right]
$$

describing the velocity and the acceleration of the target in an inertial Cartesian coordinate system $O x y z$, expressed respectively in $[\mathrm{m} / \mathrm{s}]$ and $\left[\mathrm{m} / \mathrm{s}^{2}\right]$. The course angle $\beta$ [rad] and the pitch angle $\varepsilon$ [rad] are calculated from the formulas

$$
\beta=\arctan \frac{v_{y}}{v_{x}}, \quad \varepsilon=\arctan \frac{v_{z}}{\sqrt{v_{x}^{2}+v_{y}^{2}}}
$$

where it is assumed that the target's pitch and yaw angles are equal to the pitch and yaw angles of the velocity vector $\mathbf{v}$. 
The vectors $\mathbf{v}$ and $\mathbf{w}$ are then projected onto the axes of the non-inertial coordinate system $O x^{\prime} y^{\prime} z^{\prime}$ associated with the target. The origin of the coordinate system $O x^{\prime} y^{\prime} z^{\prime}$ coincides with the initial point of the velocity vector $\mathbf{v}$. The $x^{\prime}$ axis coincides with the direction and sense of $\mathbf{v}$. The orientation of the $y^{\prime}$ and $z^{\prime}$ axes in space depends on the instantaneous values of the angles $\beta$ and $\varepsilon$. The projection of the vectors $\mathbf{v}$ and w onto the axes of the local coordinate system $O x^{\prime} y^{\prime} z^{\prime}$ is performed using the operations

$$
\left[\begin{array}{c}
v_{x}^{\prime} \\
v_{y}^{\prime} \\
v_{z}^{\prime}
\end{array}\right]=\mathbf{M}\left[\begin{array}{l}
v_{x} \\
v_{y} \\
v_{z}
\end{array}\right]=\mathbf{M v},\left[\begin{array}{l}
w_{x}^{\prime} \\
w_{y}^{\prime} \\
w_{z}^{\prime}
\end{array}\right]=\mathbf{M}\left[\begin{array}{l}
w_{x} \\
w_{y} \\
w_{z}
\end{array}\right]=\mathbf{M w}
$$

where $v_{x}^{\prime}, v_{y}^{\prime}, v_{z}^{\prime}$ and $w_{x}^{\prime}, w_{y}^{\prime}, w_{z}^{\prime}$ are the components of the velocity vector $\mathbf{v}$ and the acceleration vector $\mathbf{w}$ in the $O x^{\prime} y^{\prime} z^{\prime}$ coordinate system, expressed respectively in $[\mathrm{m} / \mathrm{s}$ ] and $\left[\mathrm{m} / \mathrm{s}^{2}\right]$, and $\mathbf{M}$ is the rotation matrix given by

$$
\mathbf{M}=\left[\begin{array}{ccc}
\cos \beta \cos \varepsilon & -\sin \beta \cos \varepsilon & \sin \varepsilon \\
\sin \beta & \cos \beta & 0 \\
-\cos \beta \sin \varepsilon & \sin \beta \sin \varepsilon & \cos \varepsilon
\end{array}\right]
$$

Finally, the roll angle $\varphi[\mathrm{rad}]$ is approximated using the formula

$$
\varphi=\arctan \frac{w_{y}^{\prime}}{g}
$$

where $g$ is the gravity acceleration $\left[\mathrm{m} / \mathrm{s}^{2}\right]$.

\section{Projectile Trajectory Reconstruction}

The position, time of arrival and velocity of the flying projectile at the point of impact are precisely measured and recorded by the acoustic locator installed onboard of the aerial target. These are the most important data for training evaluation, and the exact reconstruction of the projectile trajectory is not essential. For visualisation purposes, only the approximate trajectory of the projectile is reproduced, and the software does not take into account nuances related to atmospheric disturbances and external ballistic processes.

Reconstruction of the trajectory is carried out in an inertial Cartesian coordinate system $O x_{p} y_{p} z_{p}$. The system coincides with a plane $S_{p}$ (Fig. 2) perpendicular to the Earth's surface, passing through the points: $P_{0}=\left(x_{0}, y_{0}, z_{0}\right)$ and $P_{p f}=\left(x_{p f}, y_{p f}, z_{p f}\right)$, where $P_{0}$ describes the position of the cannon and $P_{p f}$ the coordinates of the projectile recorded by the acoustic locator, both in the $O x y z$ coordinate system defined in Section 2. The following discrete forms are thus used to describe projectile velocity and position in consecutive instants of time:

$$
\begin{gathered}
v_{p x}\left(t_{i-1}\right)=\frac{m}{m-k t_{s}} v_{p x}\left(t_{i}\right) \\
v_{p y}\left(t_{i-1}\right)=v_{p y}\left(t_{i}\right)=0 \\
v_{p z}\left(t_{i-1}\right)=\frac{m}{m-k t_{s}}\left[v_{p z}\left(t_{i}\right)+g t_{s}\right]
\end{gathered}
$$




$$
\begin{gathered}
x_{p}\left(t_{i-1}\right)=x_{p}\left(t_{i}\right)-\left(t_{s}-\frac{k}{2 m} t_{s}^{2}\right) v_{p x}\left(t_{i-1}\right) \\
y_{p}\left(t_{i-1}\right)=y_{p}\left(t_{i}\right)=0 \\
z_{p}\left(t_{i-1}\right)=z\left(t_{i}\right)+\frac{1}{2} g t_{s}^{2}-\left(t_{s}-\frac{k}{2 m} t_{s}^{2}\right) v_{p z}\left(t_{i-1}\right)
\end{gathered}
$$

where $v_{p x}, v_{p y}$ and $v_{p z}$ are the components of projectile velocity vector $\mathbf{v}_{p}[\mathrm{~m} / \mathrm{s}], x_{p}(t)$, $y_{p}(t)$ and $z_{p}(t)$ are the flat components of the projectile trajectory in the $O x_{p} y_{p} z_{p}$ system $[\mathrm{m}], m$ is the projectile mass $[\mathrm{kg}], k$ is the simplified drag proportionality coefficient $[\mathrm{kg} / \mathrm{s}], t_{s}$ is the sampling time $[\mathrm{s}]$, and $g$ is the gravitational constant $\left[\mathrm{m} / \mathrm{s}^{2}\right]$.

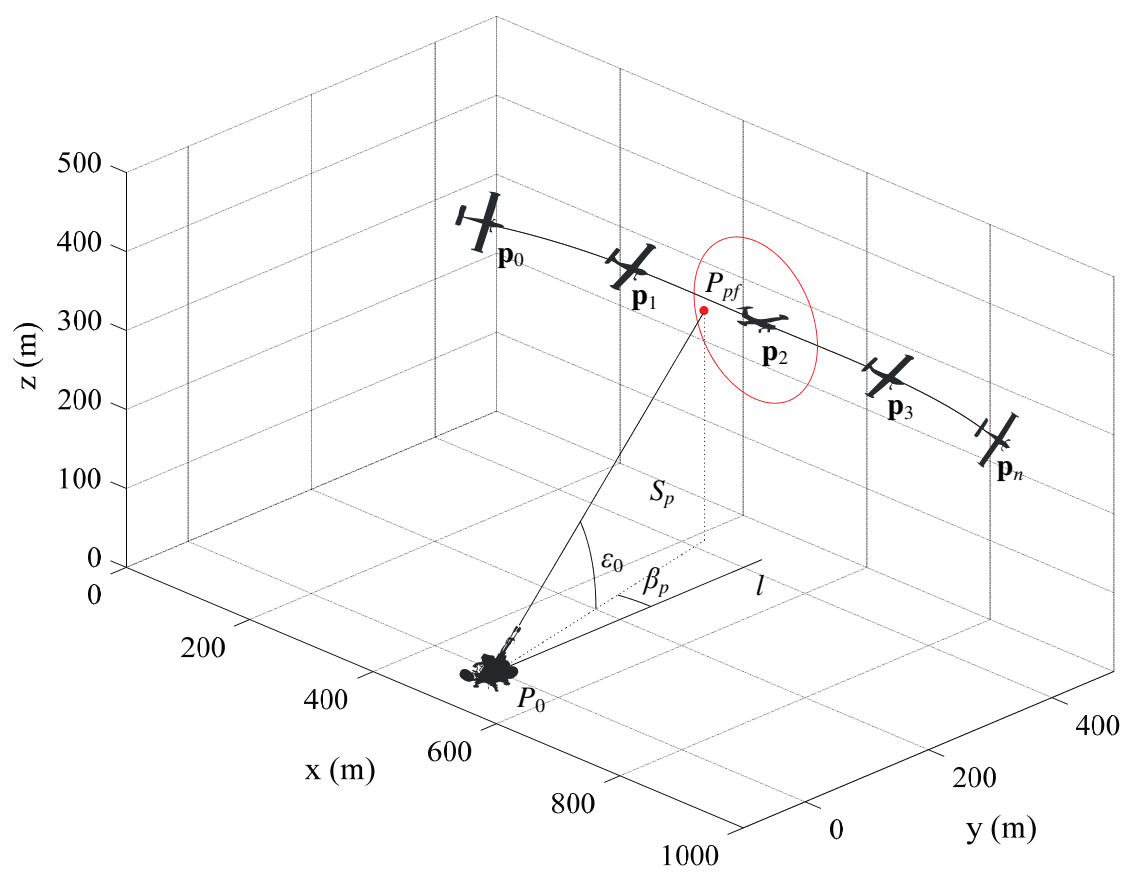

Fig. 2 Interpretation of geometrical relationships used in computational reconstruction. The detection range of the acoustic locator for point $\boldsymbol{p}_{2}$ is marked with a red circle; the detected position $P_{p f}$ of the projectile is marked with a red dot. For visualisation purposes, the scale of models and ranges has not been preserved.

As seen from the Eqs (19)-(24), trajectory reconstruction is realised via inverse calculation with respect to time. As the moment of firing is unknown, the final time $t_{p f}$ is taken as the beginning of the simulation (i.e. $t_{p f}=0$ ), and the trajectory calculations are made from the end to the beginning of the firing process (i.e. the time of shot $t_{0}$ takes place minus $n$ seconds before $t_{p f}$ ). Simulation is interrupted at the moment when the projectile estimated velocity module $v_{p}$ is equal to the velocity registered at the moment of the real shot. Initial values of $v_{p x}$ and $v_{p z}$ at point $P_{p f}$ are calculated using the angle $\varepsilon_{0}$ between line segment $P_{0} P_{p f}$ and its projection on the Earth's surface. Instantaneous values of projectile pitch angle $\varepsilon_{p}$ [rad] are approximated using the formula 


$$
\varepsilon_{p}=\arctan \frac{v_{p z}}{v_{p x}}
$$

and the azimuth angle $\beta_{p}$ [rad] is calculated as the angle included between the projection of the line segment $P_{0} P_{p f}$ on the Earth's surface and the line $l$ parallel to the $y$ axis of the system $O x y z$ (cf. Fig. 2).

Finally, the projection of the projectile trajectory onto the axes of the coordinate system $O x y z$ is performed using the rotation matrix $\mathbf{R}$ given as

$$
\mathbf{R}=\left[\begin{array}{ccc}
\cos \left(-\beta_{p}+\frac{\pi}{2}\right) & -\sin \left(-\beta_{p}+\frac{\pi}{2}\right) & 0 \\
\sin \left(-\beta_{p}+\frac{\pi}{2}\right) & \cos \left(-\beta_{p}+\frac{\pi}{2}\right) & 0 \\
0 & 0 & 1
\end{array}\right]
$$

\section{Analysis Software. Visualisation of the Training Scenario}

The specialized software supports the evaluation of anti-aircraft crews' training processes and skills improvements. It is intended to be used in the analysis and presentation of results following the completion of the training exercise. The application consists of three modules: a) visualisation, b) statistics, and c) simulation and analysis.

A modified version of the Matlab script described in [16] is used as an engine for the visualisation of firing training results. This initial outcome is used to support engineering computations and to verify the proposed solution. The final module will be implemented in the $\mathrm{C}++$ programming language, using the Qt and OpenGL libraries, allowing for portability between different system platforms and use of the system in real-time mode.

The engine is composed of several Matlab-code files providing the visualisation of solid models in 3D space. It can produce both static images and animations which can be used for creating tactical scenarios. The construction of a 3D scene is achieved by placing consecutive objects that act, in effect, like building blocks within the layout. Each of these objects is defined by a matrix of kinematic data $\mathbf{K}$ and the 3D model geometry structure $\mathbf{D}$, which are merged into one binary file. More detailed information about data organisation can be found in [16].

Matrix $\mathbf{K}$ collects all the data required by the visualisation software for the complete description of the 3D object position and orientation in successive instants of time. For the aerial target, values for filling matrix $\mathbf{K}$ are obtained from the discrete forms of flat components $x(t), y(t), z(t)$ of spatial spline $s(t)$, and from the discrete forms of $\beta(t), \varepsilon(t), \varphi(t)$, calculated using Eqs (15) and (18). For projectiles, the required values are taken as a result of approximate inverse trajectory reconstruction, described by Eqs (19)-(26). Structure D collects vertex and face lists of model geometry, which are used by the Matlab patch function. The software supports the 3D models stored in OBJ files created by popular $3 \mathrm{D}$ graphics and animation software, such as Blender or 3ds Max (Fig. 3).

Based on the visualisation engine just described, three software modules have been developed.

The visualisation module recreates the firing process, providing a visual representation of the trajectories of the projectiles and the aerial target. The aerial target is 
overlaid with multi-coloured semi-transparent circles (the virtual "shooting shield") on which markers ("bullet holes") indicate projectiles penetrating the target field (Fig. 4). In this mode, the user can select any desired camera angle, and can slow down, accelerate or pause the animation at any time. Detailed data are stored in report file, including information on target field hits, the projectile ID, the time of strike, and the distance from the centre of mass of the aerial target. The images obtained from the rendered scenes can be either captured and saved as single frames in PNG format, with user-defined resolution and dpi value, or retained as elements of the MP4 format frameset.

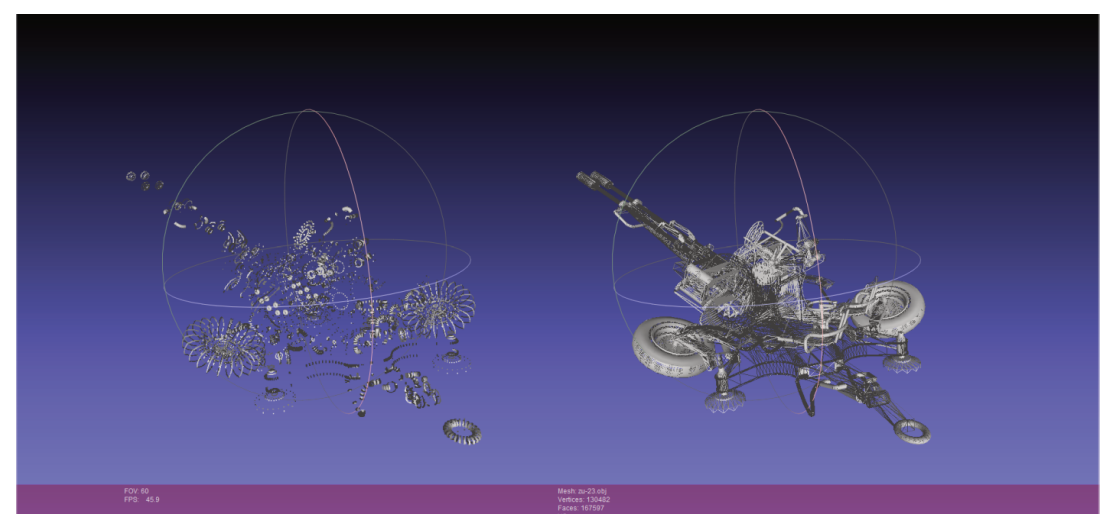

Fig. 3 Model of towed $23 \mathrm{~mm}$ anti-aircraft twin-barrelled autocannon: an example of an object supported by the software

The statistics module is used to organize overall training data in table form. The user receives information on the number of projectiles recorded for different ranges, and on the firing percentage success rate (calculated as the ratio of the number of strikes on a field with a radius less than 5 meters to the total number of projectiles detected by the acoustic locator).

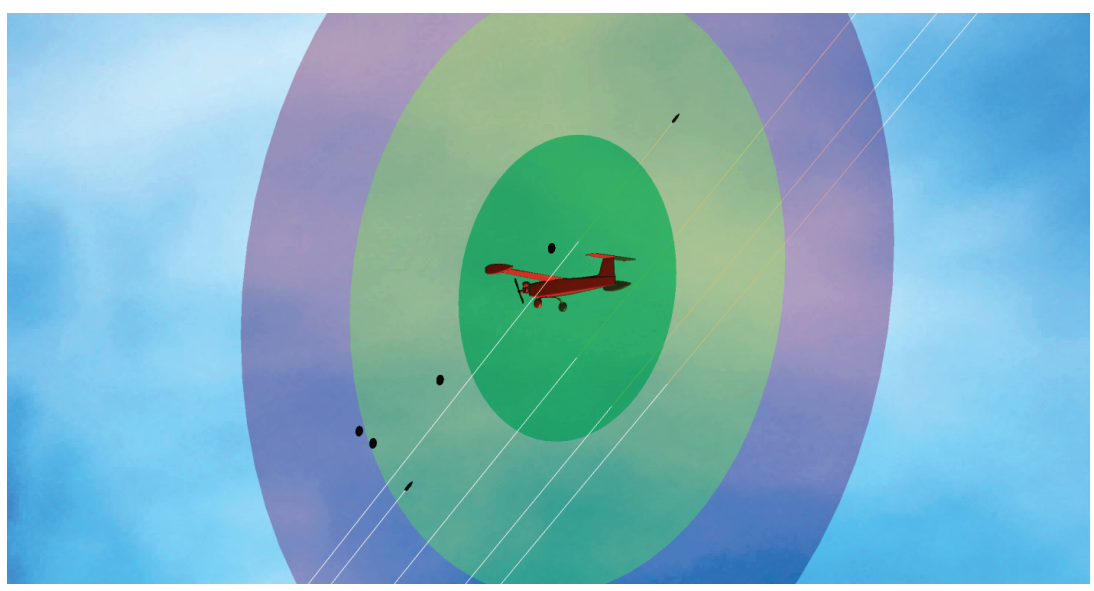

Fig. 4 Reconstruction of the training scenario 
The final element is the simulation and analysis module, which is used to simulate the effectiveness of the anti-aircraft firing carried out by the trained subunit. The user selects 3D models of aerial targets from a database (Fig. 5). In this mode, effectiveness is computed as the percentage ratio of the number of "hits" in the $3 \mathrm{D}$ virtual model to the total number of projectiles detected by the AMDI system during the firings to the real aerial target (Fig. 6).

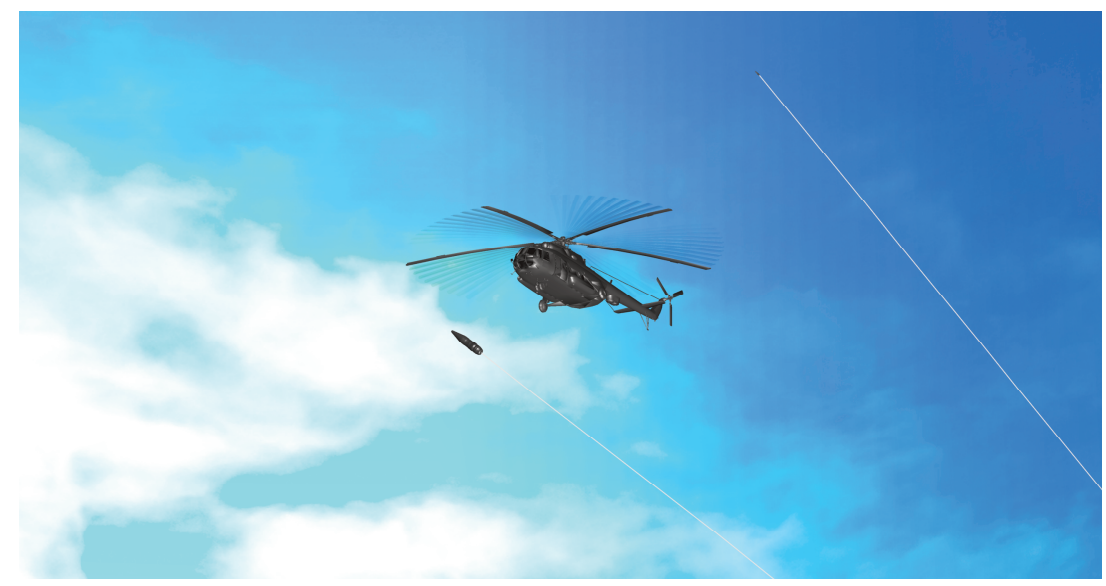

Fig. 5 Simulation and analysis module

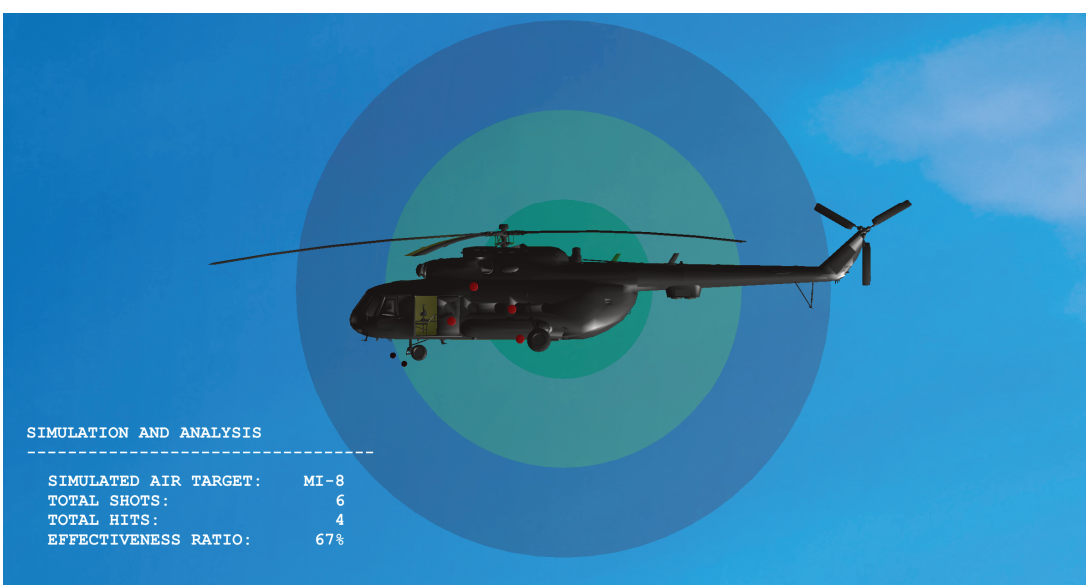

Fig. 6 Final estimated effectiveness result

\section{Conclusions and Final Remarks}

This paper describes a computational reconstruction of an anti-aircraft artillery firing scenario and its use in analysing firing data. Computational reconstruction methods supported by the use of $3 \mathrm{D}$ visualisation techniques can be a useful tool for evaluating soldiers' training levels.

The proposed method supports the precise analysis process of the final results, helping in identification of the most frequently committed errors and allowing for 
a discussion of the level of effectiveness of a particular procedure using statistical data taken from earlier firing exercises.

Another significant feature is the attractive way in which results are presented to the personnel undergoing training. Training results recorded and stored in the AMDI system database enable the reconstruction of the firing process and the creation of 3D scenes featuring the trajectories of projectiles and the aerial target. The aerial target is overlaid with multi-coloured semi-transparent circles (the virtual "shooting shield") on which markers ("bullet holes") indicate projectiles penetrating the target field. The user can select any desired camera angle, and can slow down, accelerate or pause the animation at any time.

The algorithms for reconstructing the air target and projectile trajectories were tested in real-life operating conditions and implemented in the form of a visualisation module that recreates the firing process. The software concept underwent initial testing in the MathWorks Matlab environment, using functions from the OpenGL library, a module for the display of 3D objects, and an original technique for the fast generation of the scene. The application code will ultimately be implemented in $\mathrm{C}++$, using the Qt family of portable libraries, which will enable the tool to run on various system platforms.

\section{References}

[1] DECK, L.L. An Optical Device for Rapid Measurement of the Speed, Dispersion, Attack Angle and Shock Wave of High Velocity Small Caliber Projectiles. In Proceedings of the 10th International Symposium on Ballistics. San Diego: American Defense Preparedness Association, 1987, p. 1-9.

[2] PIETRASIEŃSKI, J., RODZIK, D., GRZYWIŃSKI, S. and MIERNIK, J. Proving Ground Testing of an Anti-Aircraft Artillery Evaluation System. Problems of Mechatronics - Armament, Aviation, Safety Engineering, 2017, vol. 8, no. 3, p. 95-108, DOI 10.5604/01.3001.0010.4114.

[3] RODZIK, D., SZCZURKO, J. and BUŻANTOWICZ, W. Optical System for Anti-Aircraft Artillery Fire Training Observation. In Proceedings of the $18^{\text {th }} \mathrm{In}$ ternational Scientific-Practical Conference "Modern information and electronic technologies”, Odessa: Politehperiodika, 2017, p. 114-115.

[4] IMAGO Video Tracker Tracking and Trajectory Systems [on line]. Gatineau (QC, Canada): IMAGO Machine Vision Inc. [viewed 2020-01-09]. Available from: http://videotargettracker.com

[5] LI, D.G. and SMITH, C.L. Shot Position Measurement: A Review and Survey Report of Projectile Location Systems in the Military and Law Enforcement Agencies [Research Report]. Perth: Edith Cowan University, Australian Institute of Security and Applied Technology, 1996. 76 p. Available from: https://trove.nla.gov.au/work/15591395

[6] Defence Research \& Development Organisation - DRDO-GOI [on line]. Delhi: Ministry of Defence, Government of India. [viewed 2020-01-09]. Available from: https://www.drdo.gov.in

[7] Air Target [on line]. Kista: Air Target Sweden AB [viewed 2020-01-09]. Available from: http://www.airtarget.se 
[8] MDI Canada [on line]. Toronto: MDI-Canada Inc. [viewed 2020-01-09]. Available from: http://www.mdicanada.ca

[9] Union TSL [on line]. Bangkok: Union TSL Ltd. [viewed 2020-01-09]. Available from: http://www.utsl.co.th

[10] BOOR, C. de. A Practical Guide to Splines. New York: Springer, 2001. 348 p. ISBN 978-0-387-95366-3.

[11] BUŻANTOWICZ, W. and BEZUBIK, B. Aerial Target Flight Path Modeling using B-Spline Curves. Problems of Mechatronics - Armament, Aviation, Safety Engineering, 2018, vol. 9, no. 4, p. 21-32. DOI 10.5604/01.3001.0012.7330.

[12] GU, X., ZHANG, Y., CHEN, J. and SHEN, L. Real-Time Cooperative Trajectory Planning Using Differential Flatness Approach and B-Splines. Applied Mechanics and Materials, 2013, vol. 333-335, p. 1338-1343. DOI 10.4028/www. scientific.net/AMM.333-335.1338.

[13] ROVENSKI, V. Modeling of Curves and Surfaces with Matlab ${ }^{\circledR}$. New York: Springer, 2010. 452 p. ISBN 978-0-387-71277-2.

[14] SCHUMAKER, L.L. Spline Functions: Computational Methods. Philadelphia: SIAM, 2015. 413 p. ISBN 978-1-61-197389-1.

[15] ZHANG, C., WANG, N. and CHEN, J. Trajectory Generation for Aircraft Based on Differential Flatness and Spline Theory. In Proceedings of the 2010 International Conference on Information, Networking and Automation. Kunming: IEEE, 2010, p. V1-110-V1-114. DOI 10.1109/ICINA.2010.5636425.

[16] BUŻANTOWICZ, W. Matlab Script for 3D Visualization of Missile and Air Target Trajectories. International Journal of Computer and Information Technology, 2016, vol. 5, no. 5, p. 419-422. ISSN 2279-0764. 